\title{
A Detection Scheme for Ground Wires Detecting based on Current Shunt Principle in Distribution Lines
}

\author{
Lefeng Cheng ${ }^{1, a^{*}}$, Tao $\mathrm{Yu}^{1, \mathrm{~b}}$, Fei Guan ${ }^{2, \mathrm{c}}$ \\ ${ }^{1}$ Electric Power College, South China University of Technology, Guangzhou, 510640, China \\ ${ }^{2}$ Zhaoqing Power Supply Bureau, Zhaoqing, 526060, China \\ alefengg@126.com, btaoyu1@scut.edu.cn, 'ouanfei112@163.com
}

\begin{abstract}
Keywords: transmission line; power system; grounding wire detection; current shunt principle; distribution network

Abstract. Grounding wire detection is an important work in distribution network, especially before recover the power supply of transmission lines. Aimed at grounding detection problems, a detection scheme based on current shunt principle was proposed and before the detailed discussion of detection scheme, several factors impact the detection precision were simulated through the PSCAD/EMTDC platform, the factors include the access of transformer, branches in transmission line, common-tower transmission lines, the earth resistance and two grounding points. The simulation results show that the factors have little impact on grounding wire detection and a variable frequency should be selected. The proposed detection scheme based on current shunt principle shows a better practicability in three-phase, two-phase and single-phase grounding situation, as well as the inter-phase short circuit. The simulative researches on the influences of grounding wire detection provide a guiding significance for the actual grounding detection work.
\end{abstract}

\section{Introduction}

It's well known that before recover the power supply in distribution network, thus it is need to make sure that no grounding wires exist in transmission line, otherwise, the serious accident of "closing with grounding wire" may happen ${ }^{[1-3]}$. Aimed at problems of grounding wire detection especially the detecting precision, it is necessary to establish electromagnetic transient simulation model to make simulative analysis and the model is should be suitable for the physical characteristics of regional power network. Recently, the literatures which studied the grounding wire detection in distribution network could be summarized simply as follows. In literature [4] the protection for earth fault line selection and fault point location by $\mathrm{S}$ injecting current in neutral isolated power system was presented, in the paper, firstly a suitable high DC voltage to the faulted phase was added to form the earth fault again, and then AC current was injected to the faulted phase and exactly a detector available for detecting the injected AC signal determines the fault point, and the device based on the proposed principle had been operated successfully. In aspect of fault distance detection and location, the characteristic of faulted voltage and current was adopted to calculate and locate faults after one phase was ground ${ }^{[5,6]}$, while the literature $[7,8]$ proposed that add rectangular diagnosis signal to the fault terminal, and according to the changing of topological structure of circuit after faults, the frequency domain analysis method was used to perform fault location. These methods were rational in theory while faced with many difficulties and limitations in aspect of practicality, and had not been promoted and applied. In addition, in literature [9] an automatic fault locating algorithm based on the signal injection method was proposed and which took the signal detectors fixed on the feeders as nodes to form the feeder-network describing matrix $L$ and received the upstream detected information to form the faulty information matrix $G$, and the fault judgment matrix $P$ was calculated by the operation of $G$ and $L$. In literature [10-12] the signal injection method based on line selection and location principle was proposed and which took use of the idle voltage transformer when the faults happen to inject the AC current signal, and traced and sought the loop of injective signal in fault line to perform line selection and fault location, and based on the method, a line selection and fault location devices were designed and plenty applied in national distribution system. While the device must be worked in condition that the lines without 
outage, and the hand-held locators were used to locate the position along the lines artificially, thus cost a lot of time, and may cause a second grounding point in system during the location work, and gave rise to automatic tripping of lines.

Therefore, a simulative analysis was made in this thesis, and aimed at several factors which impacted the precision of grounding wire detection, the simulative model based on PSCAD/EMTDC was established, the factors included the access of transformer, a double transmission line on the same tower, the earth resistance, branches in line, and two grounding points, based on simulative analysis, a detection scheme based on current shunt principle was presented and which could effectively detect the single-phase, two-phase, three-phase grounding fault and inter-phase short cut. Compared with the simulative and theory analysis, it shows that the proposed scheme is in accordance with the basic theory of the method, and has a certain practicality.

\section{Electromagnetism simulation analysis}

Simulation analysis for access of transformer. The access of transformer should be taken into consideration. In actual lines maintenance, the transformer is generally access in distribution line, and according to national regulation, the transformer whose capacity over 160 MVA is often connected into Y,d11 type, and bellow 160 MVA is adopted Y,yn0 connection type, this kind of regulation is also suitable for distribution system. Therefore, for $35 \mathrm{kV}$ and $10 \mathrm{kV}$ distribution transformers, the connection type generally adopts $\mathrm{Y}, \mathrm{d} 11$; and $10 / 0.4 \mathrm{kV}$ distribution transformers adopt Y,yn0 or $\mathrm{D}, \mathrm{yn} 11$. The type of three-phase four-wire distribution mode is often put to use in our national urban and rural power grids, and for $10 \mathrm{kV}$ three-phase distribution transformer, the Y,yn0 connection mode is widely used, for it is convenient to provide $380 \mathrm{~V}$ and $220 \mathrm{~V}$ power to electricity users.

It's well known that the parameters of transformer generally including resistance, reactance, conductance and susceptance. Generally, we can obtain four data which represent the electrical properties from the builder's name plate, such as short circuit loss, short circuit voltage, no-load loss and no-load current. These data can be used to calculate the parameters of common equivalent circuits of transformer, but the parameters calculated are got under the power frequency, and if the actual situation is not power frequency, then it's need to calculate in conversion, and the formulas are shown as following (1) (4).

$$
\begin{gathered}
R_{T}=\frac{\Delta P_{S} V_{N}^{2}}{S_{N}^{2}} \times 10^{3}(\Omega) \\
X_{T} \approx \frac{U_{S} \% \cdot U_{N}^{2}}{100 S_{N}} \times 10^{3}(\Omega) \\
G_{T}=\frac{\Delta P_{F e}}{U_{N}^{2}} \times 10^{-3} \approx \frac{\Delta P_{0}}{U_{N}^{2}} \times 10^{-3}(S) \\
B_{T} \approx \frac{I_{0} \% \cdot S_{N}}{100 U_{N}^{2}} \times 10^{-3}(S)
\end{gathered}
$$

Where $S_{N}$ is rated capacity of transformer; $U_{N}$ is rated voltage of transformer; $\Delta P_{S}$ is short circuit loss; $U_{S} \%$ is percent of impedance voltage; $\Delta P_{0}$ is no-load loss; $I_{0} \%$ is percent of no-load current.

Theoretically, the neutral point in high voltage side of $10 / 0.4 \mathrm{kV}$ distribution transformer isn't connected to the earth, thus under the case of no other grounding wires exist, it cannot be formed a loop, further, no current flows through the transformer. If any other grounding wire exists, while whose three phases are installed correctly, also no current flows through transformer, only under the circumstance that, only one phase or two phases is ground connection can the current flow through transformer.

The type of transformer in the simulation is selected S11-100/10, whose capacity is $100 \mathrm{kVA}$, as is mentioned before, in China, the connection group number of $10 / 0.4 \mathrm{kV}$ transformer is generally Y,yn0, with no-load loss of $200 \mathrm{~W}$, load loss of $1500 \mathrm{~W}$, short-circuit impedance of $4.0 \%$, no-load current of 
$1.0 \%$, then the parameters of transformer module can be set based on these known data. The three-phase double winding transformer module is selected as PSCAD/EMTDC model for calculation, and the interface of parameters setting is shown in Fig.1.

The grounding wire is supposed exist in the simulation, and only one phase or two phases is electrical grounding, the frequency of injective signal is selected $60 \mathrm{~Hz}$, the length of line is taken $5 \mathrm{~km}$, and the simulative model is shown in Fig. 2.
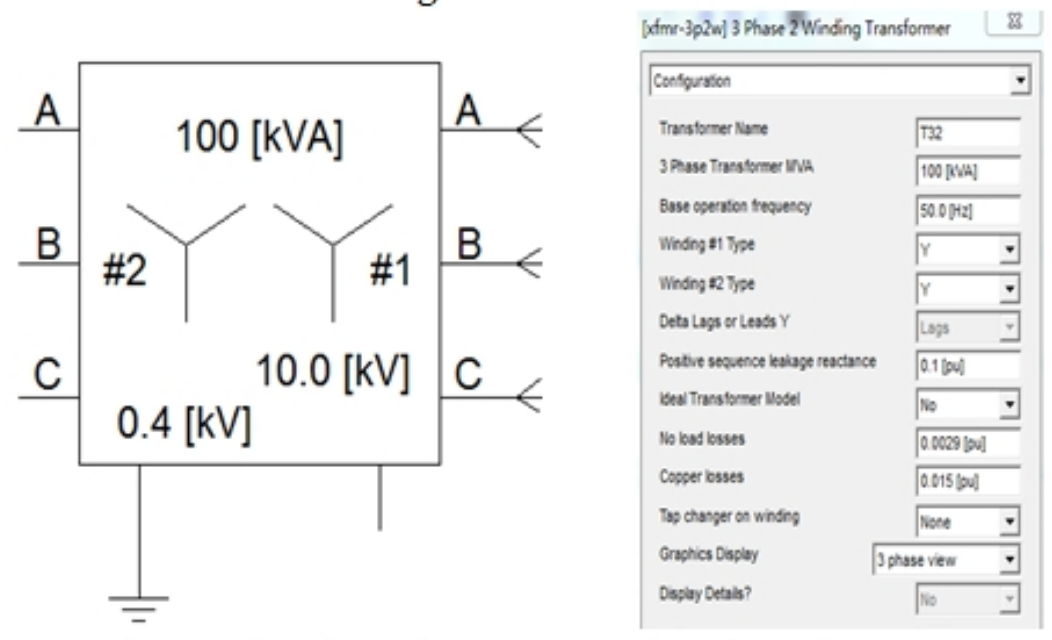

Fig.1 The interface of parameters setting of transformer

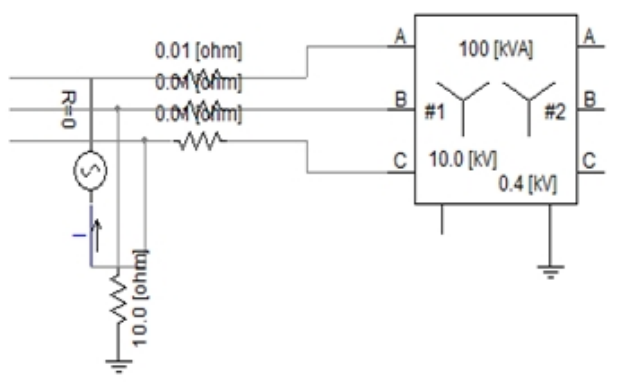

[ al the electromagnetic transient simulation model of transformer

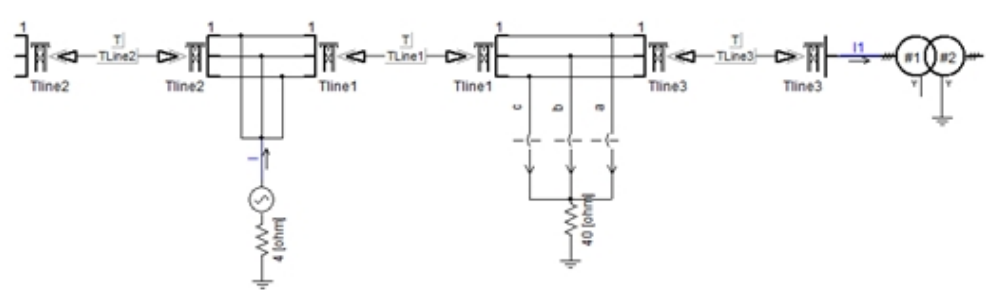

[ b] the simulative model when considering the access of transfomer

Fig.2 The simulation model when considering access of transformer in distribution network

Two cases are considered in the simulation, one is three-phase grounding, and measure the current flows through the grounding wire and transformer; the other is single-phase grounding, measure the current flows through the grounding wire and transformer, the simulative situation is shown in Fig.3.

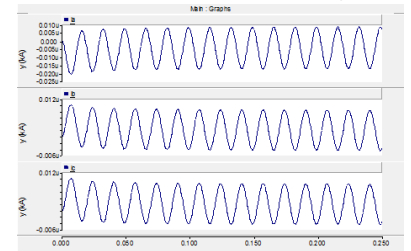

(a) The current flows through the transformer in case 1

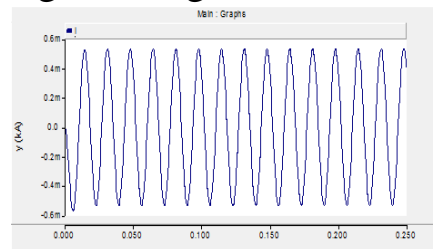

(b) The current in the grounding wire in case 1

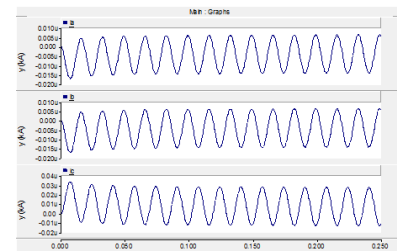

(c) The current flows through the transformer in case 2

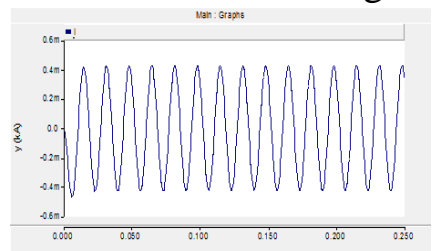

(d) The current in the grounding wire in case 2

Fig.3 The simulation results when considering access of transformer in distribution network

Seen from Fig.3 above, It's true that the current flows through the transformer, and its value, compares with the current in the grounding wire, is relatively small, and this is mainly because the impedance which is reflected by the transformer is larger, and when the signal frequency is greater, for example, the injective signal frequency is took $240 \mathrm{~Hz}$, and the simulative result is shown in Fig.4, which shows that the current is perspicuously smaller than the value under injective signal of $60 \mathrm{~Hz}$.

It's well known from the simulative diagrams that, when the single-phase or two-phase grounding condition exists, the current value flows through the transformer is explicitly smaller than the case of three-phase grounding. This is because that three-phase grounding condition is similar to three-phase lines are parallel to the ground, and when in condition of single-phase or two-phase grounding, only 
one phase or two phases can form a loop, compared with the case of three phases in parallel, the value of total impedance increases, thus the current in the grounding wire will reduce.

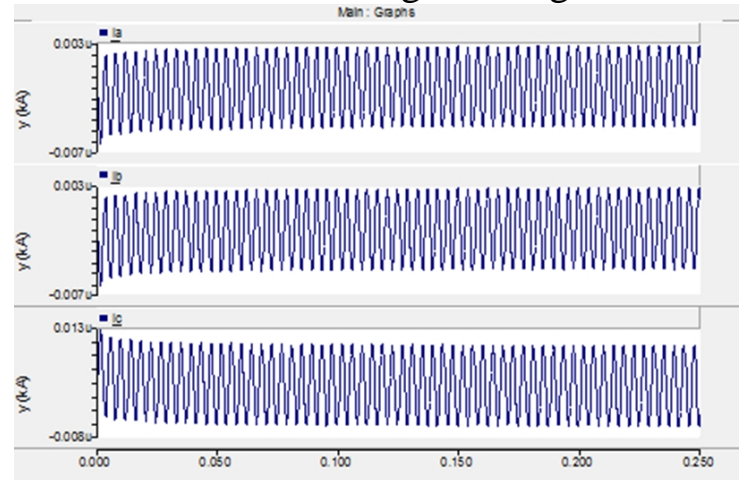

Fig. 4 The current flows through the transformer under injective signal of $240 \mathrm{~Hz}$ when a single-phase grounding condition exists

Simulation for certain branches exist in transmission line. In the actual distribution network line, which has several branches and it will have an impact on the actual measurement. In theory, as long as the signal frequency is chosen properly, the influence of distribution capacitance of the branch line can be reduced, and the length of branch line has little effect on the electric current when there are other grounding wires exist, because the current is mainly flowing through the two groups of grounding wires, therefore, the length of branch line has an impact on the current only in the case when there no grounding wires exist. Tab.1 shows the effective value statistics of current in case when there no grounding wires exist, and the length of branch line is respectively took $0,5,15,30,60$, and $80 \mathrm{~km}$.

Tab.1 The statistical table of current effective value under different length of branch lines

\begin{tabular}{cc}
\hline The length of branch lines $(\mathrm{m})$ & The effective value of current when there are no other grounding wires exist $(\mathrm{A})$ \\
\hline 0 & 0.00320 \\
5 & 0.00324 \\
10 & 0.00334 \\
30 & 0.00402 \\
60 & 0.00543 \\
80 & 0.00640 \\
\hline
\end{tabular}

As is shown in Tab.1, the statistics shows that the influence is smaller when the length of branch line is shorter, and when the length is longer, the branches are equivalent to the main backbone-branch, and the original main backbone-branch becomes the branches.

Simulation for the double transmission line on the same tower. The simulative model for the influence of double transmission line on the same tower is shown in Fig.5 (a) and the induced current is shown in Fig.5 (b). In the actual situation, the problem of double transmission line on the same tower exist based on aforementioned situation, that is, the case exists that one of the transmission line is stopped and another line is working, so the inductive disturbance signal exists, and in the absence of the grounding wire, the simulation results show that there is a higher induction voltage on a line in the outage, and the peak value researches 200V, seen from Fig.5, the amplitude of the induced current in the outage line is about $15 \mathrm{~mA}$.

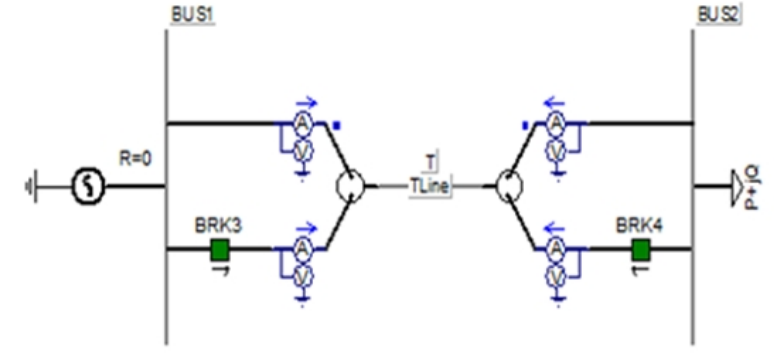

(a) the simulative model when considering double transmission line on the same tower

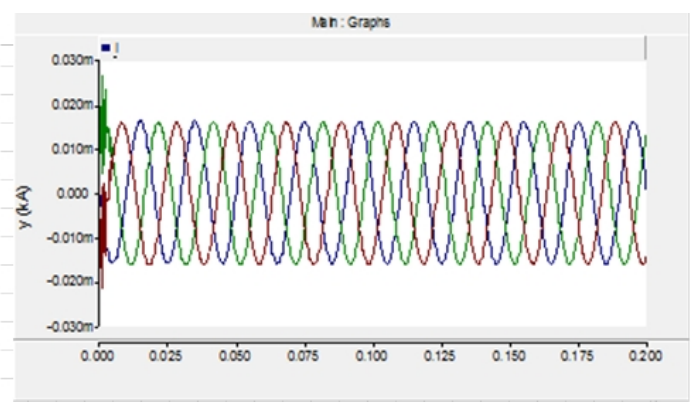

(b) the simulative wave diagram of inductive current

Fig.5 The simulative model and inductive current diagram 
Based on the previous research we can know that there is a larger induction voltage signal in the double transmission line on the same tower and in most extreme case the value researches $500 \mathrm{~V}$, the induced voltage signal may have an impact on the proposed signal injection method, and also may on the equipment and maintenance personnel. Considering that the power of induced voltage is lower, and when the transmission line is connected to the earth through a resistance, the induced voltage can be reduced a lot, thus, aimed at one phase of the transmission maintenance lines with induced voltage, the simulation is performed under different grounding resistance, and which is respectively took $100 \Omega, 1 \mathrm{k}$ $\Omega, 10 \mathrm{k} \Omega$ and $1 \mathrm{M} \Omega$, the simulative results are shown in Fig.6 (a) (h). At the same time, the central point induction voltage after three phases in short cut is close to 0 , and there is a certain induction voltage due to unbalance, which is shown in Fig.6 (i).

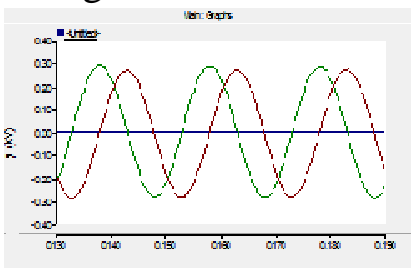

(a) The inductive voltage under $100 \Omega$ resistance

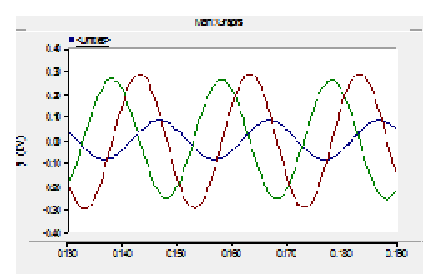

(e) The inductive voltage under $10 \mathrm{k} \Omega$ resistance

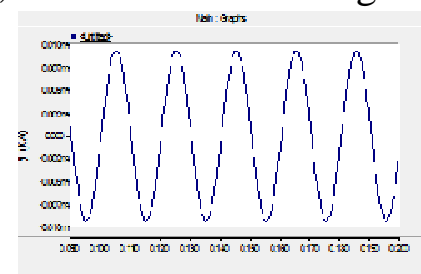

(b) The inductive current under $100 \Omega$ resistance

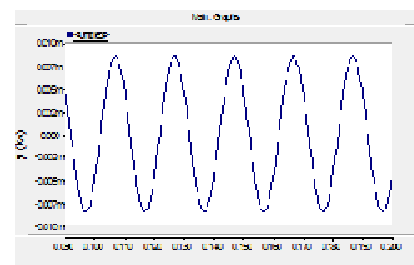

(f) The inductive current under $10 \mathrm{k} \Omega$ resistance

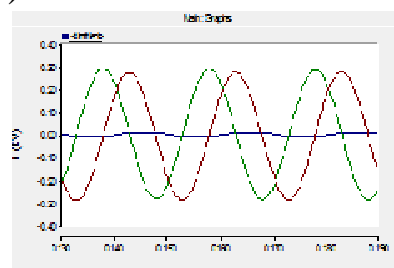

(c) The inductive voltage under $1 \mathrm{k} \Omega$ resistance

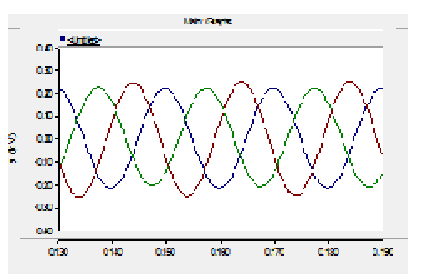

(g) The inductive voltage under $1 \mathrm{M} \Omega$ resistance

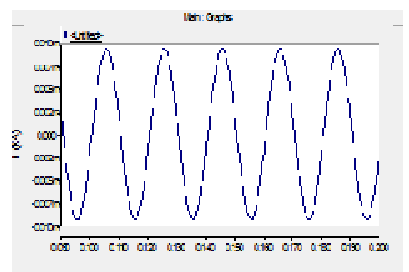

(d) The inductive current under $1 \mathrm{k} \Omega$ resistance

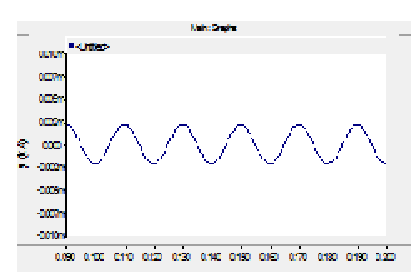

(h) The inductive current under $1 \mathrm{M} \Omega$ resistance

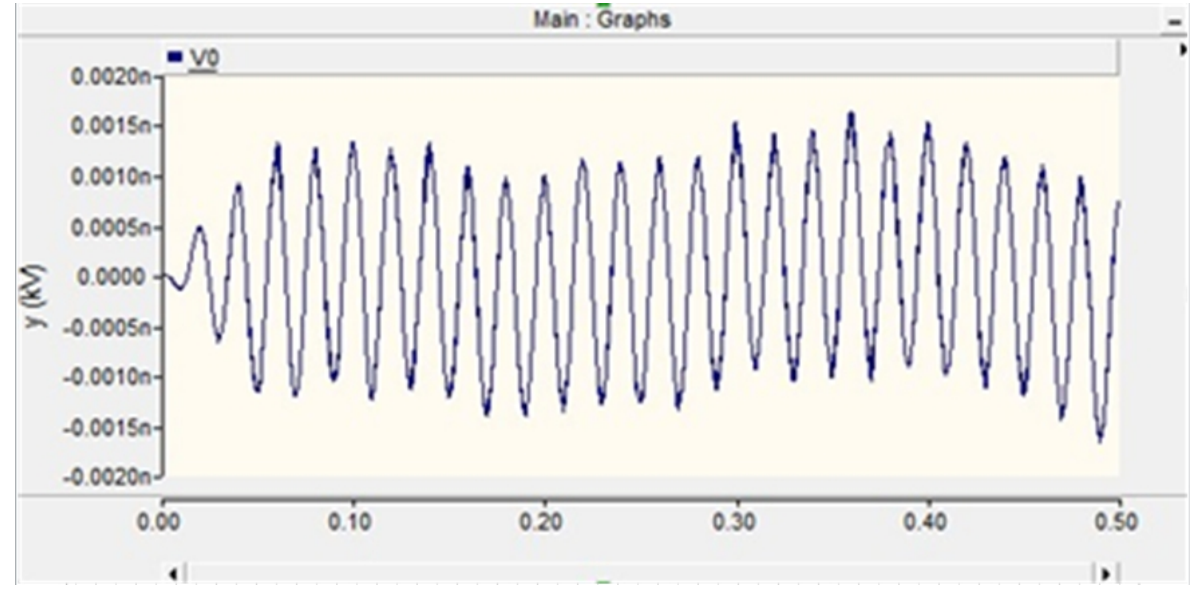

(i) The central point induction voltage after three phases in short cut Fig. 6 The simulation wave diagram under different resistance

According to the Fig.6 (i), it can be known that, the grounding is performed through the repeating resistance can not only be convenient for signal injection but also reduce the induced voltage in the line effectively by selecting a suitable value, as well as inhibit the power current in the loop circuit when the transmission line is ground, and greatly reduce the influence of interference signal to the detection accuracy, and also ensure the safety of the equipment and operators.

Simulation for and the earth resistance. It is known from the aforementioned discussion that when the specified value of earth resistance is very small, and which is not often normal in actual situation. The thesis selects $120 \mathrm{~Hz}$ as the frequency of injective signal and the length of transmission line is respectively took $5 \mathrm{~km}, 15 \mathrm{~km}, 30 \mathrm{~km}$ and $60 \mathrm{~km}$, under different simulative conditions, the earth resistances statistics are made. The effective value statistics table of current when the grounding wire exists or not exists is shown in Tab. 2 as follows. 
Seen from Tab.2, we can conclude that under the injective signal with $120 \mathrm{~Hz}$, the difference of current value in the case when the grounding wires exist and not exist is reducing, with the growing increase of length of line and grounding resistance, but we cannot distinguish the difference between the two influences, in theory, as long as the value is set in the detector, and when the detecting value of current is beyond the limited value, then we can judge that other grounding wires exist.

The above discussion is the study of injective voltage source method, the conclusion is drawn from the electromagnetic transient simulation and field test data, that is, the proposed detection method is feasible, the frequency of injective signal should be selected the medium range and especially the inter-harmonics, and the value of voltage is determined by the actual measurement precision. A threshold value of current is just set in the grounding wire and if the measuring value is greater than the set value then we can judge the existence of other grounding wire.

Tab.2 The statistical table of current effective value

\begin{tabular}{cccc}
\hline $\begin{array}{c}\text { Length of line } \\
/ \mathrm{km}\end{array}$ & $\begin{array}{c}\text { Grounding } \\
\text { resistance } / \Omega\end{array}$ & $\begin{array}{c}\text { The effective value of current in } \\
\text { the grounding wire /A }\end{array}$ & $\begin{array}{c}\text { The effective value when on } \\
\text { grounding wires exist /A }\end{array}$ \\
\hline \multirow{5}{*}{5} & 4 & 0.2817 & \\
& 50 & 0.2310 & 0.0016 \\
100 & 0.0865 & \\
200 & 0.0467 & \\
500 & 0.0242 & \\
& 4 & 0.0099 & \\
10 & 0.2415 & \\
50 & 0.2042 & \\
& 100 & 0.0835 & \\
& 200 & 0.0460 & \\
500 & 0.0240 & \\
& 4 & 0.0099 & \\
& 10 & 0.1340 & \\
& 50 & 0.1245 & \\
& 100 & 0.0714 & \\
& 200 & 0.0430 & \\
& 500 & 0.0234 & \\
& 4 & 0.0100 & \\
& 10 & 0.0664 & \\
& 50 & 0.0645 & \\
& 100 & 0.0500 & \\
& 200 & 0.0360 & \\
& 500 & 0.0219 & \\
& & 0.0101 & \\
\hline
\end{tabular}

Simulation for two grounding points. Based on theory analysis, theoretically, the symmetrical short-cut points have no current, which were correspond to directly short circuit, and no current passed through the transmission lines after the first grounding point, thus the case of two grounding points have no impacts on grounding wire detection. The simulative result in case of only one grounding point was same with the case of two grounding points.

\section{Grounding wire detection scheme based on the current shunt principle}

The principle of current shunt. In addition to the aforementioned embedded voltage source method for grounding wire detection, another detection scheme based on current shunt principle is proposed in the thesis and its specific detection principle is expounded in details next. The detection scheme is performed in the case that one group of earth wire isn't removed and based on current shunt principle and the scheme is described as follows. A small current source is connected into the line. Theoretically, in case when no other grounding wires exist, the value of current flows through the unremoved grounding wire is approximately equal to the size of the injected current source, while when other groups of grounding wires exist, the current in unremoved grounding wire will reduce due to distributary, so by comparing the current in the last unremoved group of grounding wire with the 
total injective current, we can detect whether there are other groups of grounding wires not removed. If we detect that other groups of grounding wire exist, the device will remind the maintenance personnel to find and remove them, when they are removed, the last group of grounding wire will be used to detect again to make sure that the other groups of grounding wires are already removed. After the other grounding wires are completely removed, we can remove the detector and then remove the last group of grounding wire. This measurement method is implemented after all the groups of grounding wires are hanged to the line before the maintenance work and the detector is installed near to the one group of grounding wire which will be removed in the last. One end of the detector is connected to a maintenance line, and the other end is connected with the grounding body of the grounding wire, all groups of grounding wire are removed except the last one group when the maintenance work is finished and then the detector is start for detection. In this way, the measurement method is performed in no violation of maintenance procedures and making sure the safety of measurement personnel. The simple principle of this method is shown in Fig.7 as follows.

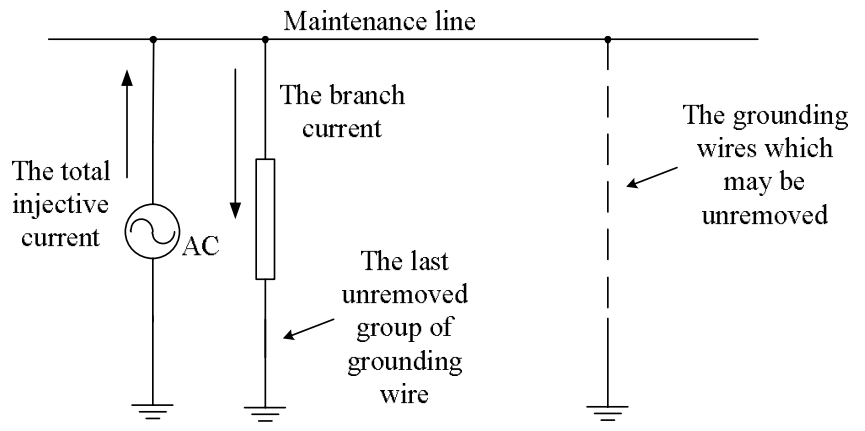

Fig.7 The simple principle of proposed detection scheme based on current shunt

Establish PSCAD/EMTDC simulation model. The constant current source is adopted in the current injection method, and in order to measure the current flows through the remaining grounding wire we can only take use of current transformer to implement measurement, thus the constant current source cannot use the DC power but the alternating current. It's well known from the aforementioned embedded voltage source detection scheme that the frequency of injective signal has an impact on the measurement due to the distributed capacitors, so after a comprehensive consideration, the signal frequency is selected $60 \mathrm{~Hz}$ will be more suitable. In this detection scheme, the factor impacts detection is the length of transmission line. Aimed at the two aspects the simulation is performed and the simulative model is shown in Fig.8.

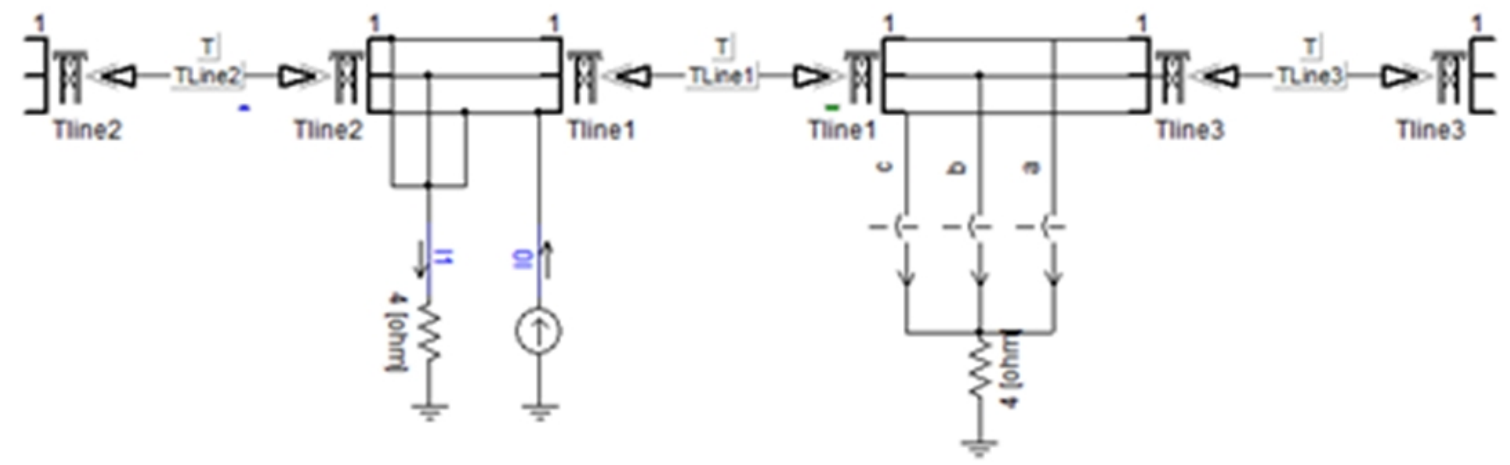

Fig.8 The PSCAD/EMTDC simulation model for the principle of detection scheme based on current shunt

When considering the influence of lines' length, suppose the grounding resistance is $4 \Omega$, the simulation results can be obtained as the Tab. 3 shows. 
Tab.3 The statistical table of current effective value

\begin{tabular}{ccc}
\hline \multirow{2}{*}{$\begin{array}{c}\text { The distance between two groups of } \\
\text { grounding wire } / \mathrm{km}\end{array}$} & \multicolumn{2}{c}{ The ratio of effective value of current $\left(I_{1} / I_{0}\right)$} \\
\cline { 2 - 3 } & $\begin{array}{c}\text { When other groups of grounding } \\
\text { wire exist }\end{array}$ & $\begin{array}{c}\text { When other groups of grounding } \\
\text { wire are removed }\end{array}$ \\
\hline 3 & 0.7704 & 1.0000 \\
\hline 15 & 0.8081 & 1.0000 \\
\hline 30 & 0.9075 & 1.0000 \\
\hline 60 & 0.9425 & 1.0000 \\
\hline 75 & 0.9591 & 1.0000 \\
\hline
\end{tabular}

We can find that from the simulative results as long as there no other groups of grounding wire exist the ratio of current value is infinitely close to 1 and when other groups of grounding wire exist, the ratio is both not 1. Although the length of the line is very long, and even the length is up to $90 \mathrm{~km}$, the ratio is only 0.9744 , as long as the detector is sufficient accurate we can get that the ratio is not 1 , then we are able to distinguish whether there are other grounding wires.

In theory, for the error grounding cases that the wire is ground in phase separation and only one phase or two phases are ground, use the detection scheme based on current shunt the error cases can be measured, and they are simulated by the model and the simulation results are shown in Tab.4, which verifies that the actual situation is accordance with the theory condition.

Of course, this method is only for reference, its disadvantages are: the constant current source needs another line, increasing the operation. But the constant current source can be installed meanwhile connecting the ground wire, and open the power switch when needs to measure, and which is removed with the last group of grounding wire together. In fact, the above two schemes proposed in the thesis have a common shortcoming, that is, aimed at the situation of three-phase short connection (that is, the interphase short circuit), the scheme is failed to detect.

Tab.4 The ratio statistical table of effective values of current

\begin{tabular}{cccc}
\hline \multirow{2}{*}{\begin{tabular}{c} 
The distance between two groups of $\begin{array}{c}\text { The ratio of effective value of current } \\
\text { grounding wire } / \mathrm{km}\end{array}$ \\
\cline { 2 - 4 }
\end{tabular}} & $\begin{array}{c}\text { Ground in phase } \\
\text { separation }\end{array}$ & $\begin{array}{c}\text { Only one phase is } \\
\text { ground }\end{array}$ & $\begin{array}{c}\text { Two phases are } \\
\text { ground }\end{array}$ \\
\hline 3 & 0.7928 & 0.8446 & 0.7945 \\
\hline 15 & 0.8213 & 0.8580 & 0.8234 \\
\hline 30 & 0.9180 & 0.9280 & 0.9131 \\
\hline
\end{tabular}

\section{Summary}

(1) The thesis firstly made simulative analysis on problems of grounding wire detection in $10 \mathrm{kV}$ distribution network, which was performed on PSCAD/EMTDC platform, and the analysis was mainly focus on impacts of several factors, which included the access of transformer, certain branches exist in transmission line, the double transmission line on the same tower, the earth resistance and two grounding points, on the detecting precision of grounding wire. From simulation results it was concluded that 1) the access of no-load transformer has litter impact on grounding point detection, because the excitation impedance of no-load transformer was very big and the current flowed which was very small; 2) the branches of transmission line had a certain impact on detection, and the higher the frequency of injective signal, the larger the error, because the grounding capacitors, and the capacitive reactance was inverse proportional to the frequency, and the detection was more affected when the capacitor reactance was in larger value. Meanwhile, the more the branches, which was equivalent to parallel, and also had a certain impact on detection; 3 ) the earth resistance had no impact on the grounding detection, because the three-phase short cut was symmetrical, and the current flowed through the resistance was zero; 4) for the double transmission line on the same tower, when a grounding wire existed, a large inductive voltage was produced in the line, and which was very small when no grounding wires existed, thus, the value of inductive voltage could be taken use to judge 
whether the grounding wires existed, while the error was must considered; 5) for two grounding points, the impact on detection could be neglected, because no current flowed through the symmetrical short-cut grounding points. 6) for the selection of frequency of injective signal, in principle, the lower the frequency, the better the detection precision, while too low of the frequency may lead to no signal to be detected, thus, a variable frequency was better to be chosen.

(2) A detection scheme based on the current shunt principle was proposed according to simulative analysis in the paper, and it was concluded that the actual measurement is consistent with the theory through the simulation and numerical statistics analysis, and the disadvantage was that the constant current source needed another wiretapping, which increased the operations, and aimed at three-phase short circuit (the inter-phase short circuit) without other grounding wires, the proposed scheme was failed to detect.

\section{References}

[1] Renqi Liu, Xiaojun Lv, Jin Huang, et al, An integrated temporary grounding line management system, J. Automation of Electric Power System (in Chinese), 2010, 34(22): 109-112.

[2] Zuoyu Zhang, Yanqing Li, A type of detecting system for grounding line, J. Power System and Clean Energy (in Chinese), 2010, 26(12): 17-23.

[3] Tao Ji, Yongduan Xue, Tongjing Sun, et al, Fault location for distribution feeders based on traveling waves, J. Automation of Electric Power System (in Chinese), 2005, 29(19): 66-71.

[4] Huifen Zhang, Zhencun Pan, Zaizhong Sang, Injecting current based method for fault location in neutral isolated power system, J. Automation of Electric Power System (in Chinese), 2004, 28(3): 64-66.

[5] Zili Peng, Lanfang Peng, Port ratio branch location of short fault for tree type power networks, J. Journal of Electronics (in Chinese),1990, 12(6), 641-645.

[6] Hongliang Gao, Xuechang Yang, Location algorithm for line-to-ground fault on power distribution lines, J. Journal of Tsinghua University (Sci \& Tech) (in Chinese), 1999, 39(9): 33-36.

[7] Guiye Yin, Xuechang Yang, Zhensheng Wu, Analyzing the criteria of transfer function algorithm for locating ground fault in power distribution networks, J. Automation of Electric Power System (in Chinese), 2000, 24(19): 29-33.

[8] Zhensheng Wu, Xuechang Yang, Guiye Yin, Analysis in frequency domain for locating ground fault in the branches of power distribution networks, J. Automation of Electric Power System (in Chinese), 2001, 25(23): 32-36, 48.

[9] Huifen Zhang, Fan Zhang, Zhencun Pan, Automatic fault locating algorithm based on signal injection method for distribution system, J. Electric Power Automation Eequipment (in Chinese), 2008, 28(6): 39-43.

[10]Zaizhong Sang, Zhencun Pan, Lei Li, "S signal injection" and fault line identifying and distance measuring and fault point locating technique , J. Proceedings of the EPSA (in Chinese), 1998, 10(4): 35-39.

[11]Zaizhong Sang, Zhencun Pan, Lei Ding, The principle and application of "S Injection Method", J. Electric Power (in Chinese), 1997, 30(6): 44-45.

[12]Zaizhong Sang, Huifen Zhang, Zhencun Pan, et al, Protection for single to earth fault line selection for ungrounded power system by injecting signal, J. Automation of Electric Power Systems (in Chinese), 1996, 20(2): 11-12, 35. 